\title{
Migration or Residency? The Evolution of Movement Behavior and Information Usage in Seasonal Environments
}

\author{
Allison K. Shaw ${ }^{\star}$ and Iain D. Couzin \\ Department of Ecology and Evolutionary Biology, Princeton University, Princeton, New Jersey 08544a \\ Submitted March 21, 2012; Accepted August 21, 2012; Electronically published November 27, 2012 \\ Online enhancement: appendixes.
}

\begin{abstract}
Migration, the seasonal movement of individuals among different locations, is a behavior found throughout the animal kingdom. Although migration is widely studied at taxonomically restricted levels, cross-taxonomic syntheses of migration are less common. As a result, we lack answers to broad questions such as what ultimate factors generally drive animal migration. Here we present such a synthesis by using a spatially explicit, individual-based model in which we evolve behavior rules via simulations under a wide range of ecological conditions to answer two questions. First, under what types of ecological conditions can an individual maximize its fitness by migrating (vs. being a resident)? Second, what types of information do individuals use to guide their movement? We show that migration is selected for when resource distributions are dominated more by seasonality than by local patchiness, and residency (nonmigratory behavior) is selected for when the reverse is true. When selected for, migration evolves as both a movement behavior and an information usage strategy. We also find that different types of migration can evolve, depending on the ecological conditions and availability of information. Finally, we present empirical support for our main results, drawn from migration patterns exhibited by a variety of taxonomic groups.
\end{abstract}

Keywords: individual-based model, information usage, migration, movement, resource heterogeneity, seasonality.

\section{Introduction}

Migration is used by a number of species as a strategy for coping with seasonally variable environments. It has long been accepted that organisms should exhibit this strategy only when it is advantageous (Lack 1954) and that the costs and benefits of migrating depend on ecological conditions (MacArthur 1959). Furthermore, at least within birds, it is believed that the machinery for migration evolved in an early ancestor and is now present across all

\footnotetext{
* Corresponding author. Present address: Division of Evolution, Ecology and Genetics, Research School of Biology, Australian National University, Canberra, Australian Capital Territory 0200, Australia; e-mail: allison.shaw@anu .edu.au.

Am. Nat. 2013. Vol. 181, pp. 114-124. (C) 2012 by The University of Chicago. 0003-0147/2013/18101-53738\$15.00. All rights reserved.

DOI: $10.1086 / 668600$
}

lineages (Berthold 1999), such that populations currently evolve to be migrants or residents mainly as a function of their present ecological conditions (Alerstam et al. 2003; Salewski and Bruderer 2007). (In this article, we use the term "evolution" in the sense of the maintenance and modification of a trait, not its first appearance; Zink 2002.) However, we still lack a synthesis of what specific types of ecological conditions select for migration, across all migratory species. This is due primarily to a lack of crosstaxonomic communication on the topic of animal migration (Bauer et al. 2009). A handful of empirical studies have used careful manipulations (e.g., Olsson et al. 2006; Brodersen et al. 2008; Grayson and Wilbur 2009) or extensive cross-species comparisons (Levey and Stiles 1992; Chesser and Levey 1998; Boyle and Conway 2007) to tease apart the factors that drive migration in a species or a small taxonomic group. Our aim is to gain an understanding of the ecological drivers more broadly. To do so, we take a theoretical approach. Surprisingly, especially given the large amount of work done on migration, there are very few simple models in the literature aimed at understanding the factors that drive the evolution of animal migration (Fryxell et al. 2011; but see Guttal and Couzin 2010; Torney et al. 2010; Holt and Fryxell 2011).

Existing general models exploring why animals migrate have focused primarily on how migratory and nonmigratory individuals can coexist within a single partially migratory population (e.g., Cohen 1967; Lundberg 1987; Kaitala et al. 1993; Taylor and Norris 2007; Griswold et al. 2010; Shaw and Levin 2011) rather than determining the ecological conditions favoring migration in the first place. Alexander (1998) estimated the costs and benefits of migration in terms of survival and growth rate for species that swim, walk, or fly to move. A more recent model (Holt and Fryxell 2011) determined the conditions favoring residency or migration, assuming no cost to migration, and a model by Wiener and Tuljapurkar (1994) showed that negative correlation between two patches selects for movement between them. However, each of these models 
considers space only implicitly and assumes migrants move between two discrete locations. Since migration is an adaptive response to resources that are heterogeneously distributed in space and time (Cresswell et al. 2011), spatially explicit models may provide insight that spatially implicit ones cannot. A number of spatially explicit models have been developed, most of which are designed to understand migration patterns in a particular population of a given species (e.g., Hubbard et al. 2004; Carr et al. 2005; Barbaro et al. 2009; Holdo et al. 2009; but see Guttal and Couzin 2010, 2011).

To our knowledge, no simulation model has tried to map out which resource distributions select for migration. This is probably due, at least in part, to the difficulty of defining migration in terms of a single behavioral parameter that can be evolved across a simulation. While no single definition of migration is agreed upon, most definitions of the term refer to both a physical movement as well as a behavioral pattern of information usage: migration is persistent, directed movement which relocates individuals on a greater scale and longer duration than normal daily activity and is a round-trip movement that often occurs seasonally (Dingle and Drake 2007). Here, we develop an individual-based model to determine what ecological conditions favor migration over residency. In "Methods," we describe our model, which consists of a resource distribution (see "Ecological Conditions") and individuals whose movement is guided by different sources of information ("Individual Behavior"). We quantify an individual's fitness in several ways ("Fitness Functions") and use a genetic algorithm to evolve individual behavior over the course of a simulation ("Selection"). We use our model to answer two questions, as described in "Results." First, what types of ecological conditions select for migratory behavior versus resident behavior ("Residency or Migratory Behavior")? Second, what types of information (resource, historical, or social) do individuals use to guide their movement and what happens if not all sources of information are available ("Information Availability")? Finally, we discuss empirical support for, and implications of, our findings with respect to both the conditions favoring migration and the information availability.

\section{Methods}

Our model consists of a spatially explicit patchy resource distribution and individuals with movement rules (fig. 1), each described in more detail below. Migration is a roundtrip movement usually between two locations (although it can take several generations to complete, as in insects). Often migration in each direction is driven primarily by a different ecological condition (see "Ecological Conditions" below), each of which would be represented by a different fitness function (see "Fitness Functions" below). Instead of simulating all possible types of round-trip migration, we only simulate movement of one leg of a migration (since movement in the reverse direction is conceptually the same).

Note that the phrase "evolution of migration" is used to refer to two distinct aspects: the first-ever appearance of migration in a lineage and the current-day maintenance of migration (Zink 2002). In the first case, the question is, how did a nonmigratory species evolve the complex suite of machinery required for migration (e.g., navigation, energy stores)? In the second case, which is the one we consider, the question is, given that a lineage has evolved the machinery it needs to migrate, under what ecological conditions is migration favored?

\section{Ecological Conditions}

Animal migration can be driven by food and water availability; escape from harsh climatic conditions, predators, parasites, and disease; and factors related to reproduction such as mate availability, nesting sites, and juvenile survival (Heape 1931; Dingle 1996). Many of these factors come into play at some point during migration, although movement in each direction is often driven by a single factor. For example, many migratory birds (in both hemispheres) move between high-latitude breeding grounds and lowlatitude wintering grounds (Jahn et al. 2004), such that pole-ward movement is driven by both food and reproduction and equator-ward movement is driven by increased winter survival. Most baleen whales also feed at high latitudes but migrate to low latitudes to reproduce (Lockyer and Brown 1981). Here, pole-ward movement is driven by food and equator-ward movement by reproduction and survival while calving (Corkeron and Connor 1999). Migratory ungulates (e.g., wildebeest) are driven by continuously changing food resources and, as a result, move in a circuit following the changing food gradient (table 2 in Harris et al. 2009; Holdo et al. 2009).

Our simulated resource distribution represents any ecological factor that could potentially drive migration (e.g., distribution of food, temperature, or nesting sites). The total resource distribution (fig. $1 A$ ) consists of a linear trend in resource availability (fig. $1 B$ ) plus a superimposed patchy resource distribution (fig. 1C). This is meant to represent any sort of resource gradient that individuals might migrate along, including latitudinal (e.g., some birds and butterflies), altitudinal (e.g., some mammals and birds), or salinity (e.g., some fish and crustaceans) gradients. The resource is defined by three parameters: the slope of the linear trend $\psi$ and the quality $p_{\mathrm{q}}$ and average width $p_{\mathrm{w}}$ of the patch distribution. If the direction of the trend reverses over the course of a year, $\psi$ can be consid- 
(A)

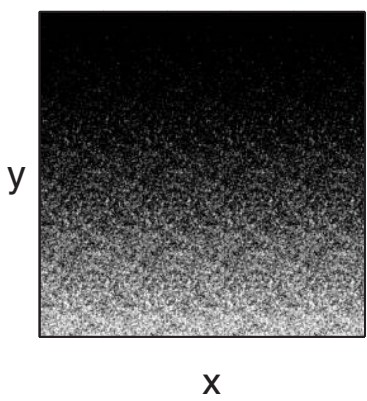

(D)

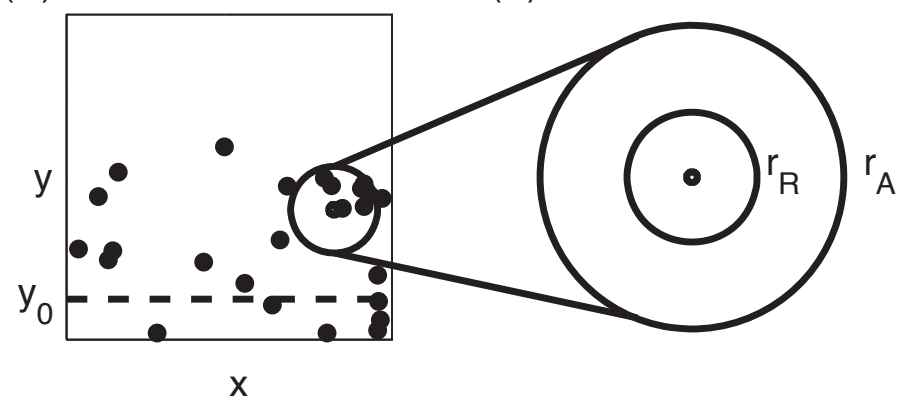

(B)

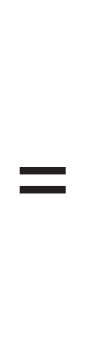

(E)

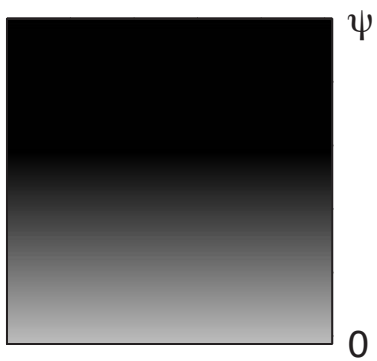

$(\mathrm{F})$

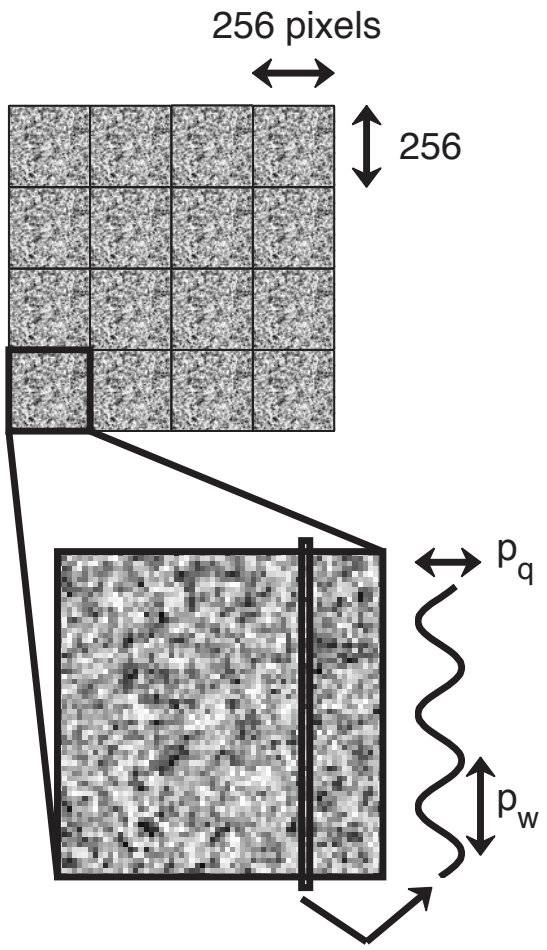

Figure 1: Schematic of the model components, which includes a heterogeneously distributed resource $(A-C, F)$ and moving individuals $(D-E)$. The resource $(A)$ is the sum of a linear trend in resources of slope $\psi(B)$ plus a patchy resource distribution of quality $p_{\mathrm{q}}$ and average width $p_{\mathrm{w}}(C, F)$, where darker indicates higher resource quality. Individuals move across the resource distribution $(D)$, driven in part by the position and velocity of other individuals within a small repulsion radius $r_{\mathrm{R}}$ and attraction radius $r_{\mathrm{A}}(E)$. Note that this schematic is for illustrative purposes and is not to scale. See "Ecological Conditions" of text for details.

ered a measure of the degree of seasonality in the resource (the difference in average resource abundance in a single area between the high-abundance season and the lowabundance season). This is the scenario we consider in this article. The patch quality $p_{\mathrm{q}}$ and patch width $p_{\mathrm{w}}$ are measures of how patchy the resource is. A high value of $p_{\mathrm{q}}$ corresponds to a resource that has high-quality patches present year-round regardless of season, and so $p_{\mathrm{q}}$ is a measure of how buffered the resource is against seasonality. Finally, the patch width $p_{\mathrm{w}}$ is a measure of average habitat patch size. We varied each of these three parameters to generate a range of different ecological conditions (see zoomed-in snapshots of the resource shown in figs. $2 A$, $\mathrm{A} 3 \mathrm{~A}, \mathrm{~A} 4 \mathrm{~A}$, available online).

We used an algorithm for the creation of colored (correlated in space and time) noise (García-Ojalvo et al. 1992), to create an initial resource patch distribution, and updated it every 100 steps (to allow patch location to change over the simulation, since actual resource patches are not static). Due to the computational constraints of simulating such a large field, we simulated a $256 \times 256-$ pixel square (where 1 pixel $=0.78$ body length $[\mathrm{BL}]$ ) shown in figure $1 F$ and tiled it to get the overall field $(1,024 \times 1,024)$ shown in figure $1 C$ (the patch distribution has periodic boundaries such that tiling does not introduce discontinuities). This tiling should not affect the overall results since individual step size is small compared to the tile size.

\section{Individual Behavior}

"Migration" is usually defined at the individual level in terms of both a movement pattern (relocation on a large spatial scale, compared to normal daily movement) and an information usage strategy (persistent, directed movement). We chose to encode individual behavior in terms of information usage (instead of movement pattern) - in our simulations, individuals were able to use three types of information to direct their movements: resource, historical, and social, given by unit vectors $\mathbf{R}, \mathbf{H}$, and $\mathbf{S}$, respectively. The resource vector $\mathbf{R}$, calculated analytically (García-Ojalvo et al. 1992; Torney et al. 2011), gave the direction of highest local resource increase from the perspective of an individual's specific location, representing 
(A)

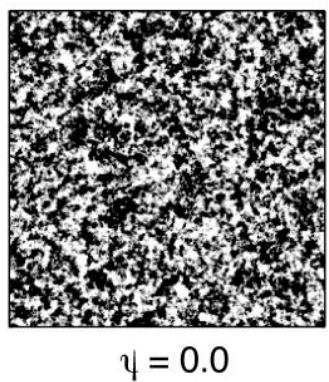

(B)

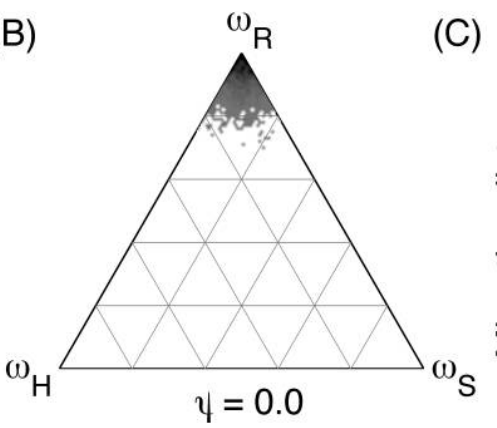

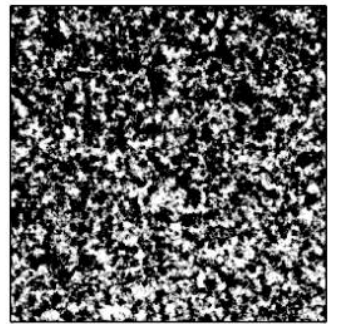

$\psi=0.02$

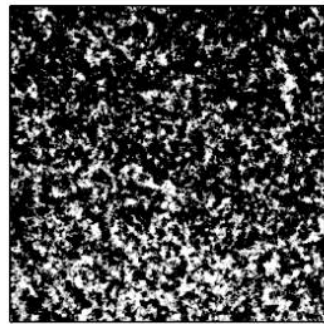

$y=0.04$

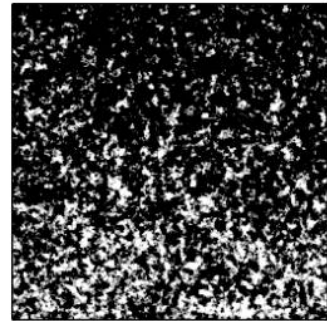

$\psi=0.05$ 
Simulated individuals move in two-dimensional space with constant speed. Each individual is characterized by a position, a velocity vector, and a set of information preference weights $\left(\omega_{\mathrm{H}}, \omega_{\mathrm{S}}\right.$, and $\left.\omega_{\mathrm{R}}\right)$, which direct their movement over the course of the simulation (fig. 1D). Each individual begins with a random $X$ coordinate in $[0,1]$ and $Y$ coordinate in $y_{0} \pm N /(2 \rho)$, where $N$ is the number of individuals and $\rho$ is the initial density. Individuals move by amount $\Delta y$ each step (set to be $0.1 \mathrm{BL}$ ), in the direction given by their velocity vector, for a total of $T$ steps per generation. The value of $T$ was chosen so that individuals cannot cross the entire space during the course of one generation. Migration is typically a temporary behavior held for one time period and followed by a second nonmigratory period. However, since residents are essentially nonmigratory for both time periods, the difference between residents and migrants occurs during the first time period. For simplicity, in our model we only simulate the first period, instead of trying to simulate two periods with a switch point in between them, which would double the number of evolving parameters.

\section{Fitness Functions}

The costs and benefits of migration can manifest themselves in a number of ways. For some species, the benefit of migration comes from the resources accumulated along the way. For example, ungulates that feed as they migrate (e.g., wildebeest, Connochaetes taurinus) derive benefit from the accumulation of resources as they move, often foraging so much that they significantly deplete the local plant biomass as they move through an area (McNaughton 1976). In other species, the benefit of migration comes in the final location. For example, Norwegian spring-spawning herring (Clupea harengu) adults migrate as far south as possible before spawning, since temperature is the main factor determining larval survival (Slotte and Fiksen 2000). Finally, for other species the cost of migration is the risk of mortality during the journey. For example, in many migratory songbirds (e.g., Catharus thrushes), the cost of migration is due to coping with cold temperatures along the migratory journey, a cost that can be higher than the extra energy expenditure from sustained flight (Wikelski et al. 2003).

To account for this variety of costs and benefits, we ran simulations with three types of fitness functions: cumulative, end-point, and minimum. For the cumulative fitness function, an individual's fitness was calculated as the sum of the values of resource it passed through at every time step over the course of a generation (to mimic the ungulate continuously foraging scenario). For the endpoint fitness function, an individual's fitness was calculated as the value of the resource at its final position at the end of a generation (to mimic a fish migrating to spawn scenario). For the minimum fitness function, an individual's fitness was calculated as the lowest resource value it passed through within a generation (for example, to mimic a song bird surviving through harsh conditions).

\section{Selection}

We evolved the $\omega$ values of individuals across many generations within an evolutionary simulation. At the start of a simulation, each of $N$ individuals was assigned a random value for $\omega_{\mathrm{H}}, \omega_{\mathrm{S}}$, and $\omega_{\mathrm{R}}$ between 0 and 1 (weights were then evenly normalized to sum to 1 ). At the end of each generation, individuals were selected to pass their strategy $\left(\omega_{\mathrm{H}}, \omega_{\mathrm{S}}\right.$, and $\omega_{\mathrm{R}}$ values), with some small mutation rate (a Gaussian random number with mean 0 and standard deviation $\mu$ ) to individuals in the next generation. Individuals were selected with replacement (a single individual could be selected more than once) where the probability of an individual being selected was proportional to its fitness. Each simulation was run for $G$ generations, where each generation was run for $C$ copies of $T$ steps each. (See table Al for all parameters and values.) For each copy of a generation, the resource distribution was regenerated (with the same parameter values) and individuals were assigned new random starting positions (described above). This was done to ensure that differences in fitness between individuals were due primarily to differences in their parameter values rather than due to differences in their random starting positions.

\section{Results}

For each set of ecological parameter values (seasonality, patch quality, and patch width), we quantified the behavior that evolved after many generations in two ways: in terms of the information usage strategy ( $\omega$ values) and in terms of the movement behavior (total distance traveled along the $Y$-axis, the direction of increasing $\psi$ ). Overall, two distinct types of behavior emerged. In the first case, individuals evolved to move almost entirely based on resource information $\left(\omega_{\mathrm{R}} \approx 1\right)$ and to ignore historical and social information (figs. $2 B, A 2-A 4$ ). These individuals essentially did not move along the $Y$-axis (figs. $2 C$, A2A4), and so we refer to these as "residents." In the second case, all individuals within a population evolved to rely to some extent on historical information $\left(\omega_{\mathrm{H}}>0\right.$; figs. $2 D$, A2-A4), where the specific value of $\omega_{H}$ depended on the ecological conditions and the fitness function used (see below). These individuals traveled very far along the $Y$ axis (figs. 2C, A2-A4), and so we refer to these as "migrants" (since their behavior was both directed and resulted in a relocation on a large spatial scale). Distances 
shown are normalized such that the maximum distance an individual could travel during the simulation is set to be 1 .

\section{Residency or Migratory Behavior}

Whether simulated individuals evolved to be residents or migrants depended on the values of ecological parameters $\psi$ (seasonality), $p_{\mathrm{q}}$ (local patch quality), $p_{\mathrm{w}}$ (local patch width) and also on the fitness metric used (cumulative, end-point, or minimum). When patchiness $\left(p_{\mathrm{q}}\right.$ and $\left.p_{\mathrm{w}}\right)$ is high compared to seasonality $(\psi)$, individuals evolve to be residents, whereas when $p_{\mathrm{q}}$ and $p_{\mathrm{w}}$ are low compared to $\psi$, individuals evolve to be migrants (figs. $2 C, \mathrm{~A} 2-\mathrm{A} 4$ ). This is true for all three fitness functions, although the parameter values at which the shift from resident to migratory behavior occurs differs. The one exception is that under the end-point fitness function, high levels of $p_{\mathrm{w}}$ do not select for residency (fig. A4C). Also, for simulations with the minimum fitness function, migration occurs only when patchiness is essentially nonexistent and the resource increases approximately monotonically up the $Y$-axis (fig. $\mathrm{A} 3 D)$.

The range of ecological conditions under which migration was favored depended in large part on the main factor driving migration (the fitness function used). Migration occurred under the broadest conditions for end-point fitness (e.g., migration to a breeding site), followed by cumulative fitness (e.g., foraging migratory ungulate), then minimum fitness (e.g., song bird surviving through harsh conditions). We confirmed these results by deriving the conditions under which migration should be favored over residency in a simple analytic model (see app. B, "Analytic Model," available online). We find that migration should be favored under an end-point fitness if

$$
\psi \Delta y T>\delta_{\text {res }},
$$

which is true under a broader range of conditions (values of $\psi$ and $\delta_{\text {res }}$ ) than the conditions favoring migration under a cumulative fitness

$$
\psi \Delta y \frac{(T+1)}{2}>\delta_{\mathrm{res}}
$$

where $\psi, \Delta y$, and $T$ have the same meaning as in our simulation model and $\delta_{\text {res }}$ is the average patch quality that a resident encounters, assuming it can seek out good patches. This makes intuitive sense-in our admittedly extreme, end-point fitness scenario, migrants are not affected at all by conditions along their journey and are therefore not disrupted by patchiness as easily. This is most clearly demonstrated by the fact that high values of $p_{\mathrm{w}}$ did not select for residency in simulations with the end-point fitness (fig. A4C).

\section{Information Availability}

Arguably organisms that had never migrated before would not have access to historical information. To see what would happen in this scenario, we ran simulations where individuals were able to use only social and resource information. We find that for low $\psi$, individuals do not migrate and for high $\psi$ individuals migrate far, as before (fig. 3, solid line). However, migrating individuals were not able to travel as far as during migrations where they could use historical information (fig. $2 C$ vs. fig. 3 ), and the information usage pattern differed slightly. For low $\psi$ (fig. 3, region I) all individuals within a population evolve to have $\omega_{\mathrm{R}} \approx 1$ and $\omega_{\mathrm{S}} \approx 0$, indicating a high reliance on resource information, and almost no reliance on social information. For intermediate $\psi$ (fig. 3, region II) all individuals evolve to have fairly high $\omega_{\mathrm{S}}$ values, indicating a higher reliance on social information when migrating. For high $\psi$ (fig. 3, region III), individuals evolve to have $\omega_{\mathrm{R}} \approx 1$ and $\omega_{\mathrm{S}} \approx 0$ but were still traveling far in the $Y$ direction. Taken together, these results suggest that without access to historical information, migratory individuals will rely on social information only when it allows them to travel further than they could based on local resource information alone (fig. 3, dashed line).

Even individuals that had migrated previously may not

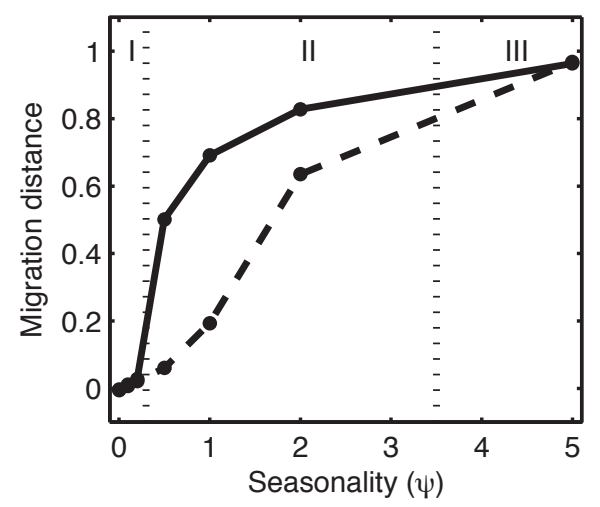

Figure 3: For intermediate seasonality, social individuals can travel farther than asocial ones, when neither type has access to historical information. Shown is the movement behavior (distance traveled) that evolved in environments with different values of $\psi$ and constant values of $p_{\mathrm{q}}(10)$ and $p_{\mathrm{w}}(8 \mathrm{BL})$, where no historical information was available and individuals could use either both social and resource information (solid line) or just resource information (dashed line). For low $\psi$ (region I), individuals evolved to have $\omega_{\mathrm{S}} \approx 0$; for intermediate $\psi$ (region II), individuals evolved to have high $\omega_{\mathrm{s}}$; and for high $\psi$ (region III), individuals evolved to have $\omega_{\mathrm{S}} \approx 0$. 
be able to perfectly remember the migratory direction. To determine what would happen in this scenario, we ran simulations where the vector $\mathbf{H}$ varied in reliability (fig. $4 A$ ). This represents a situation where historical information is either remembered imperfectly (e.g., individuals are constrained in their memory abilities) or where information is not a good indicator of resource distributions (e.g., if the best resource location is not consistent from one year to the next). We find that when $\mathbf{H}$ was very reliable (low $\sigma$ ), individuals relied on $\mathbf{H}$ to direct their migrations $\left(\omega_{\mathrm{H}} \approx 1, \omega_{\mathrm{S}} \approx 0\right.$, and $\left.\omega_{\mathrm{R}} \approx 0\right)$ and when $\mathbf{H}$ was not reliable (high $\sigma$ ), individuals relied more on $\mathbf{S}$ to migrate (fig. $4 B$ ). This suggests that as the quality of historical information deteriorates, migratory individuals would be expected to rely more heavily on social information instead.

\section{Discussion}

Although migration is a well-studied phenomenon, surprisingly there are relatively few models that seek to explain the ecological conditions under which migration should be favored. Here we present such a model, in the form of an individual-based simulation where individuals move across a spatially explicit patchy resource distribution, guided by a number of different information sources. We evolve each individual's strategy, defined by the relative weight values $\left(\omega_{\mathrm{H}}, \omega_{\mathrm{S}}\right.$, and $\left.\omega_{\mathrm{R}}\right)$ that it gives to each source of information (historical, social, and resource), under a variety of ecological conditions and fitness functions in order to determine what conditions select for migratory behavior, and what information individuals use to guide their movement.

We quantified our results in terms of both the values of evolved parameters, and the movement behavior of individuals with those parameter values. Two distinct behavior types emerged in the simulations. "Residents" predominantly use resource information to direct their movement and, as a result, tend not to travel far in the $Y$ direction. "Migrants" primarily use nonresource (historical or social) information and as a result traveled far in the $Y$ direction. This result confirms the concept that migration corresponds to both a change in information usage behavior (temporarily ignoring local resources) and also physical movement (traveling relatively long distances).

The type of behavior (resident or migrant) that simulated individuals evolved depended on the spatial distribution of resources-some resource distributions selected for residency behavior and others selected for migratory behavior. In our model, the benefit of migration comes from an increase in average local resources (determined by $\psi$ ), and the cost of migration comes from the locally poor resource patches (determined by $p_{\mathrm{q}}$ and $p_{\mathrm{w}}$ ) that the individual passes through during the migration. Migration occurred only in a seasonal environment $(\psi>0)$, and within seasonal environments, migration evolved if $p_{\mathrm{q}}$ and
(A)
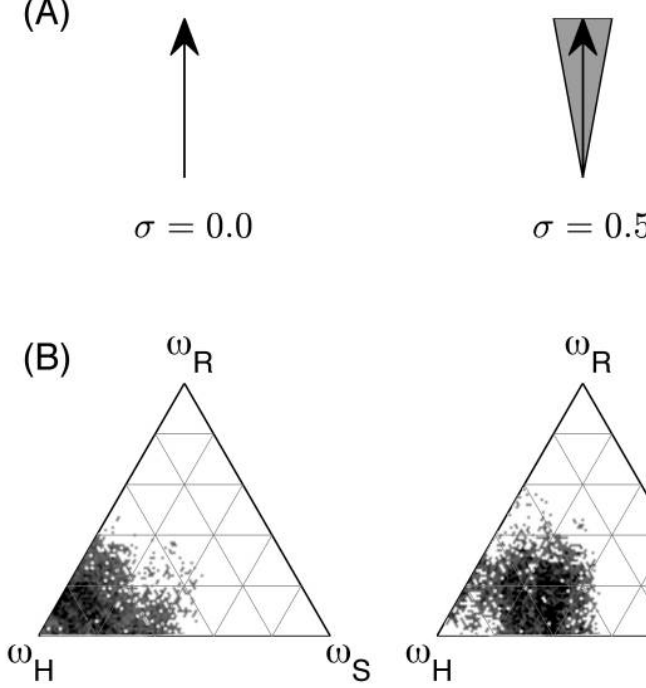

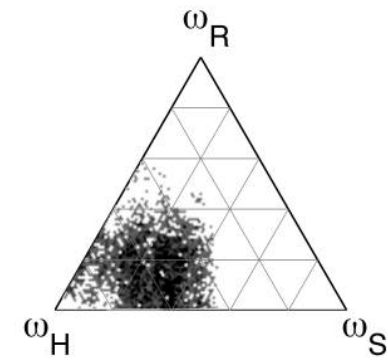

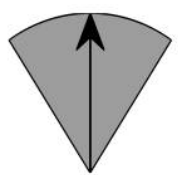

$\sigma=1.0$

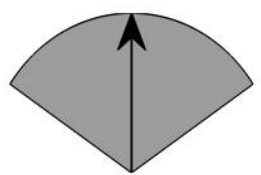

$\sigma=2.0$

Figure 4: Individuals shift to rely more on social information during migration as historical information becomes more inaccurate. Information usage $(B)$ that evolved in environments with constant values of $\psi(0.2), p_{\mathrm{q}}(10)$, and $p_{\mathrm{w}}(8 \mathrm{BL})$, but with different accuracy levels of historical information, $\mathbf{H}$, as shown in $A$. Ternary plots are shown in $B$, where each dot shows the three evolved $\omega$ values of a single individual in the population; corners correspond to behavior dominated by the indicated $\omega$ parameter, and each panel shows the result of a simulation with a different value of $\sigma$. 
$p_{\mathrm{w}}$ were low compared to $\psi$ and the benefits of migrating outweighed the costs, and residency evolved if the reverse was true-a straightforward result that can be confirmed analytically (see app. B). The ratio of $\psi$ to $p_{\mathrm{q}}$ and $p_{\mathrm{w}}$ is similar to the concept of a signal-to-noise ratio. In our model, we found a sharp transition between those ecological conditions that select for migration and those that select for residency, although this result seems to be model specific (see app. B).

In our model we simulate the migratory movement of one leg of a migration, instead of a full round-trip migration, since each migration leg may be characterized by a different region of parameter space. For the return leg of migration to occur, the general conditions must be reversed, such that the location with lower-quality resources in one season becomes the location with higher-quality resources the next season and vice versa.

For species whose migration is driven by the same resource in each direction (e.g., food) or by different but correlated factors in each direction (e.g., food and temperature), our results predict that highly seasonal environments should select for migration. This result is supported by comparative studies across species in European birds (Herrera 1978), North American birds (Newton and Dale 1996), raptors (Kerlinger 1989), and bats (Fleming and Eby 2003), and across populations within a species in striped bass, Morone saxatilis (Coutant 1985). Our results also predict that seasonal nonbuffered resources should select for migration, while seasonal buffered resources should select for residency. Which particular resource this result applies to (food, temperature, breeding sites, etc.) depends on the species. Extensive comparative studies on Neotropical birds match our predictions-species living in unbuffered open habitats and feeding on fruit tend to migrate, while those in more buffered habitats (forest interior; feeding on insects) tend to be residents (Levey and Stiles 1992; Chesser and Levey 1998; Boyle and Conway 2007). Bell (2011) also found that while migration frequency in North American passerines generally increases with latitude (due to increased resource seasonality), the variance in this trend can be explained by residency being more common in species that rely on buffered resources. Similarly, temperate bat species that roost in open trees are more likely to migrate than cave-roosting bats, since caves offer a more buffered (constant temperature) environment during harsh winters (Popa-Lisseanu and Voigt 2009). Finally, our results predict that migration should be more common in seasonal environments with smaller habitat patches-a prediction that has been supported in white-tailed deer (Odocoileus virginianus), where individuals in areas with large average forest patch size were less likely to migrate (Grovenburg et al. 2011).

For some species, migration is driven by different factors in each direction, the most common example being species that migrate between feeding site and spawning sites (e.g., Shaw and Levin 2011). In this case $\psi$ is no longer a measure of seasonality but rather a measure of how much better it is, on average, to breed at site A than at site B and to feed at site B than at site A. Here, migration is expected to occur in species where the best reproduction and feeding habitats are in different locations. This prediction matches migration patterns in a number of species, including baleen whales, which migrate between high-latitude feeding grounds and low-latitude breeding grounds (Corkeron and Connor 1999); land crabs, which migrate from terrestrial feeding areas to aquatic breeding areas (Wolcott and Wolcott 1985); and diadromous fish, which move between freshwater and saltwater, based on which area has higher productivity (catadromy in the tropics and anadromy in temperate regions; Gross et al. 1988).

While we have so far only discussed migration as a seasonal (annual) event, the results of our model could be applied to organismal movement across a broader range of temporal scales. For example, if $\psi$ is interpreted as resource fluctuations on a daily timescale, our model matches the observation that daily fluctuations in light levels are necessary for zooplankton daily vertical migration to occur (Dodson 1990). On the other end of the time spectrum, if $\psi$ is interpreted on the order of thousands of years, our model matches the observation that glacial-interglacial periods seem to force patterns of forest migration (McGlone 1996).

In our model, we focus on the conditions that select for migration to be maintained in a population (assuming individuals have the necessary migratory machinery) and not on the conditions that first selected for this machinery. This separation of timescales is a reasonable assumption for birds (Berthold 1999; Alerstam et al. 2003; Salewski and Bruderer 2007), but at this time it is unknown to what extent it holds in other taxonomic groups. Additionally, migration is thought to interact with a number of other life-history factors such as body size (Roff 1988) and mating systems (García-Peña et al. 2009), which we do not explicitly consider in our model.

When all three sources of information (historical, social, resource) were available to individuals, migrants relied primarily on historical information. If historical information was either unavailable or inaccurate, migrants relied on social and resource information-essentially pooling their knowledge of local resource conditions via social interactions in order to migrate (the "many wrongs principle"; Simons 2004). This suggests that a population with no previous history of migration could establish migratory behavior through extended social behavior. However, once the migratory route is learned (if possible), individuals should rely on this new historical information rather than 
social interactions, since in our simulations migrants that relied on historical information traveled longer distances and had higher fitness than migrants relying on only social information (fig. $2 \mathrm{C}$ vs. fig. 3). A couple of individualbased models have previously shown that decreasing the number of individuals in the group with historical knowledge about the direction of travel (e.g., older individuals) can drastically change the ability of the group to migrate (Huse et al. 2002; Couzin et al. 2005).

Within migratory populations in which the direction of highest resource changes frequently or is unreliable, we expect that individuals would rely more heavily on social rather than historical information. Unfortunately this is currently difficult to test since little is known about the relative importance of historical, environmental, and social cues in migratory species (Brown and Laland 2003; Noordwijk et al. 2006). Since our main interest was determining what behavior evolved given certain constraints on information availability, we did not allow the historical vector (H) to evolve during our simulations. Recent work suggests that if the accuracy of $\mathbf{H}$ can be improved at a cost (relative to obtaining social information), there is a strong information-based frequency dependence, where some individuals evolve to invest in highly accurate $\mathbf{H}$ and are then exploited by other individuals in the population (Guttal and Couzin 2010; Torney et al. 2010).

Here we have presented an individual-based simulation model designed to determine what types of ecological conditions select for migration. We derive a number of predictions, which are all supported by examples from a number of different taxonomic groups. The creation of such a general model has two main benefits. First, it allows us to conduct essentially an extended thought experiment and test ideas that would be difficult to test empirically. Second, by keeping the model generic and generating predictions that can be tested in a number of species, we can draw parallels across a variety of taxonomic groups, which we hope will inspire further cross-taxonomic comparisons of migratory patterns.

\section{Acknowledgments}

A.K.S. thanks J. Chapman, C. Jørgensen, J. Metcalfe, T. Piersma, E. Rees, S. Bauer, and other members of the "Animal Migration-Linking Models and Data" Lorentz Center Workshop for conversations that helped inspire the manuscript. We also thank F. Bartumeus, S. Levin, D. Rubenstein, L. Salvador, D. Stanton, C. Staver, C. Torney, and members of the Couzin lab for help with the model and manuscript development. To simulate the resource distribution, we used code written by C. Torney. This material is based upon work supported by the National Science
Foundation (NSF) Graduate Research Fellowship under grant DGE-0646086 to A.K.S. I.D.C. gratefully acknowledges support from by NSF grant PHY-0848755 (to I.D.C.), the Searle Scholars Program (to I.D.C.), Office of Naval Research grant N00014-09-1-1074 (to I.D.C.), Army Research Office grant W911NG-11-1-0385 (to I.D.C.), and Defense Advanced Research Projects Agency grant HR0011-09-1-0055 (to Princeton University).

\section{Literature Cited}

Alerstam, T., A. Hedenström, and S. Åkesson. 2003. Long-distance migration: evolution and determinants. Oikos 103:247-260.

Alexander, R. M. 1998. When is migration worthwhile for animals that walk, swim or fly? Journal of Avian Biology 29:387-394.

Aoki, I. 1982. A simulation study on the schooling mechanism in fish. Bulletin of the Japanese Society of Scientific Fisheries 48:10811088.

Barbaro, A., B. Einarsson, B. Birnir, S. Sigurðsson, H. Valdimarsson, Ó. Pálsson, S. Sveinbjörnsson, and P. Sigurðsson. 2009. Modelling and simulations of the migration of pelagic fish. ICES Journal of Marine Science 66:826-838.

Bauer, S., Z. Barta, B. J. Ens, G. C. Hays, J. M. McNamara, and M. Klaassen. 2009. Animal migration: linking models and data beyond taxonomic limits. Biology Letters 5:1-4.

Bell, C. P. 2011. Resource buffering and the evolution of bird migration. Evolutionary Ecology 25:91-106.

Berthold, P. 1999. A comprehensive theory for the evolution, control and adaptability of avian migration. Ostrich 70:1-11.

Boyle, W. A., and C. J. Conway. 2007. Why migrate? a test of the evolutionary precursor hypothesis. American Naturalist 169:344359.

Brodersen, J., P. A. Nilsson, L. Hansson, C. Skov, and C. Brönmark. 2008. Condition-dependent individual decision-making determines cyprinid partial migration. Ecology 89:1195-1200.

Brown, C., and K. N. Laland. 2003. Social learning in fishes: a review. Fish and Fisheries 4:280-288.

Carr, S. D., J. L. Hench, R. A. Luettich Jr., R. B. Forward Jr., and R. A. Tankersley. 2005. Spatial patterns in the ovigerous Callinectes sapidus spawning migration: results from a coupled behavioralphysical model. Marine Ecology Progress Series 294:213-226.

Chesser, R. T., and D. J. Levey. 1998. Austral migrants and the evolution of migration in New World birds: diet, habitat, and migration revisited. American Naturalist 152:311-319.

Cohen, D. S. 1967. Optimization of seasonal migratory behavior. American Naturalist 101:5-17.

Corkeron, P. J., and R. C. Connor. 1999. Why do baleen whales migrate? Marine Mammal Science 15:1228-1245.

Coutant, C. C. 1985. Striped bass, temperature, and dissolved oxygen: a speculative hypothesis for environmental risk. Transactions of the American Fisheries Society 114:31-61.

Couzin, I. D., J. Krause, N. R. Franks, and S. A. Levin. 2005. Effective leadership and decision-making in animal groups on the move. Nature 433:513-516.

Cresswell, K. A., W. H. Satterthwaite, and G. A. Sword. 2011. Understanding the evolution of migration through empirical examples. Pages 7-16 in E. Milner-Gulland, J. M. Fryxell, and A. R. 
Sinclair, eds. Animal migration: a synthesis. Oxford University Press, New York.

Dingle, H. 1996. Migration: the biology of life on the move. Oxford University Press, New York.

Dingle, H., and V. A. Drake. 2007. What is migration? BioScience 57:113-121.

Dodson, S. 1990. Predicting diel vertical migration of zooplankton. Limnology and Oceanography 35:1195-1200.

Fleming, T. H., and P. Eby. 2003. Ecology of bat migration. Pages 156-208 in T. H. Kunz and M. B. Fenton, eds. Bat ecology. University of Chicago Press, Chicago.

Fryxell, J. M., E. Milner-Gulland, and A. R. Sinclair. 2011. Introduction. Pages 1-3 in E. Milner-Gulland, J. M. Fryxell, and A. R. Sinclair, eds. Animal migration: a synthesis. Oxford University Press, New York.

García-Ojalvo, J., J. M. Sancho, and L. Ramírez-Piscina. 1992. Generation of spatiotemporal colored noise. Physical Review A 46: 4670-4675.

García-Peña, G. E., G. H. Thomas, J. D. Reynolds, and T. Szekely. 2009. Breeding systems, climate, and the evolution of migration in shorebirds. Behavioral Ecology 20:1026-1033.

Grayson, K. L., and H. M. Wilbur. 2009. Sex-and context-dependent migration in a pond-breeding amphibian. Ecology 90:306-312.

Griswold, C. K., C. M. Taylor, and D. R. Norris. 2010. The evolution of migration in a seasonal environment. Proceedings of the Royal Society B: Biological Sciences 277:2711-2720.

Gross, M. R., R. M. Coleman, and R. M. McDowall. 1988. Aquatic productivity and the evolution of diadromous fish migration. Science 239:1291-1293.

Grovenburg, T. W., C. N. Jacques, R. Klaver, C. DePerno, T. Brinkman, C. Swanson, and J. Jenks. 2011. Influence of landscape characteristics on migration strategies of white-tailed deer. Journal of Mammalogy 92:534-543.

Guttal, V., and I. D. Couzin. 2010. Social interactions, information use, and the evolution of collective migration. Proceedings of the National Academy of Sciences of the USA 107:16172-16177.

- 2011. Leadership, collective motion and the evolution of migratory strategies. Communicative and Integrative Biology 4 : 294-298.

Harris, G., S. Thirgood, J. G. C. Hopcraft, J. P. G. M. Cromsigt, and J. Berger. 2009. Global decline in aggregated migrations of large terrestrial mammals. Endangered Species Research 7:55-76.

Heape, W. 1931. Emigration, migration and nomadism. Heffer, Cambridge.

Herrera, C. M. 1978. On the breeding distribution pattern of European migrant birds: MacArthur's theme reexamined. Auk 95: 496-509.

Holdo, R. M., R. D. Holt, and J. M. Fryxell. 2009. Opposing rainfall and plant nutritional gradients best explain the wildebeest migration in the Serengeti. American Naturalist 173:431-445.

Holt, R. D., and J. M. Fryxell. 2011. Theoretical reflections on the evolution of migration. Pages 17-31 in E. Milner-Gulland, J. M. Fryxell, and A. R. Sinclair, eds. Animal migration: a synthesis. Oxford University Press, New York.

Hubbard, S., P. Babak, S. T. Sigurdsson, and K. G. Magnússon. 2004. A model of the formation of fish schools and migrations of fish. Ecological Modelling 174:359-374.

Huse, G., S. Railsback, and A. Feronö. 2002. Modelling changes in migration pattern of herring: collective behaviour and numerical domination. Journal of Fish Biology 60:571-582.
Huth, A., and C. Wissel. 1992. The simulation of the movement of fish schools. Journal of Theoretical Biology 156:365-385.

Jahn, A. E., D. J. Levey, and K. G. Smith. 2004. Reflections across hemispheres: a system-wide approach to New World bird migration. Auk 121:1005-1013.

Kaitala, A., V. Kaitala, and P. Lundberg. 1993. A theory of partial migration. American Naturalist 142:59-81.

Katz, Y., K. Tunstrøm, C. Ioannou, C. Huepe, and I. D. Couzin. 2011. Inferring the structure and dynamics of interactions in schooling fish. Proceedings of the National Academy of Sciences of the USA 108:18720-18725.

Kerlinger, P. 1989. Flight strategies of migrating hawks. University of Chicago Press, Chicago.

Lack, D. 1954. The significance of migration. Pages 243-254 in The natural regulation of animal numbers. Oxford University Press, Oxford.

Levey, D. J., and F. G. Stiles. 1992. Evolutionary precursors of longdistance migration: resource availability and movement patterns in Neotropical landbirds. American Naturalist 140:447-476.

Lockyer, C. H., and S. G. Brown. 1981. The migration of whales. Pages 105-137 in D. J. Aidley, ed. Animal migration. Cambridge University Press, Cambridge.

Lukeman, R., Y. X. Li, and L. Edelstein-Keshet. 2010. Inferring individual rules from collective behavior. Proceedings of the National Academy of Sciences of the USA 107:12576-12580.

Lundberg, P. 1987. Partial bird migration and evolutionarily stable strategies. Journal of Theoretical Biology 125:351-360.

MacArthur, R. 1959. On the breeding distribution pattern of North American migrant birds. Auk 76:318-325.

McGlone, M. S. 1996. When history matters: scale, time, climate and tree diversity. Global Ecology and Biogeography Letters 5:309-314.

McNaughton, S. J. 1976. Serengeti migratory wildebeest: facilitation of energy flow by grazing. Science 191:92-94.

Mueller, T., and W. F. Fagan. 2008. Search and navigation in dynamic environments-from individual behaviors to population distributions. Oikos 117:654-664.

Newton, I., and L. C. Dale. 1996. Bird migration at different latitudes in eastern North America. Auk 113:626-635.

Noordwijk, A. J., F. Pulido, B. Helm, T. Coppack, J. Delingat, H. Dingle, A. Hedenström, et al. 2006. A framework for the study of genetic variation in migratory behaviour. Journal of Ornithology 147:221-233.

Olsson, I. C., L. A. Greenberg, E. Bergman, and K. Wysujack. 2006. Environmentally induced migration: the importance of food. Ecology Letters 9:645-651.

Partridge, B. L., and T. J. Pitcher. 1980. The sensory basis of fish schools: relative roles of lateral line and vision. Journal Of Comparative Physiology 135:315-325.

Popa-Lisseanu, A. G., and C. C. Voigt. 2009. Bats on the move. Journal of Mammalogy 90:1283-1289.

Reynolds, C. W. 1987. Flocks, herds and schools: a distributed behavioral model. Pages 25-34 in Proceedings of the 14th Annual Conference on Computer Graphics and Interactive Techniques. Association for Computing Machinery, New York

Roff, D. A. 1988. The evolution of migration and some life-history parameters in marine fishes. Environmental Biology of Fishes 22: 133-146.

Salewski, V., and B. Bruderer. 2007. The evolution of bird migration—a synthesis. Naturwissenschaften 94:268-279. 
Shaw, A. K., and S. A. Levin. 2011. To breed or not to breed: a model of partial migration. Oikos 120:1871-1879.

Simons, A. M. 2004. Many wrongs: the advantage of group navigation. Trends in Ecology \& Evolution 19:453-455.

Slotte, A., and O. Fiksen. 2000. State-dependent spawning migration in Norwegian spring-spawning herring. Journal of Fish Biology 56:138-162.

Taylor, C. M., and D. R. Norris. 2007. Predicting conditions for migration: effects of density dependence and habitat quality. Biology Letters 3:280-283.

Torney, C., A. Berdahl, and I. D. Couzin. 2011. Signaling and the evolution of cooperative foraging in dynamic environments. PLoS Computational Biology 7:e1002194.

Torney, C., S. A. Levin, and I. D. Couzin. 2010. Specialization and evolutionary branching within migratory populations. Proceedings of the National Academy of Sciences of the USA 107:20394-20399.
Warburton, K., and J. Lazarus. 1991. Tendency-distance models of social cohesion in animal groups. Journal of Theoretical Biology 150:473-488.

Wiener, P., and S. Tuljapurkar. 1994. Migration in variable environments: exploring life-history evolution using structured population-models. Journal of Theoretical Biology 166:75-90.

Wikelski, M., E. Tarlow, A. Raim, R. Diehl, R. Larkin, and G. Visser. 2003. Costs of migration in free-flying songbirds. Nature 423:704.

Wolcott, T. G., and D. L. Wolcott. 1985. Factors influencing the limits of migratory movements in terrestrial crustaceans. Contributions in Marine Science 68:257-273.

Zink, R. M. 2002. Towards a framework for understanding the evolution of avian migration. Journal of Avian Biology 33:433-436.

Associate Editor: Edmund D. Brodie III

Editor: Judith L. Bronstein 